This document is the Accepted Manuscript version of a Published Work that appeared in final form in J. Chem. Phys. 131, 024119 (2009), copyright $(\subset)$ American Institute of Physics after peer review and technical editing by the publisher. To access the final edited and published work see

jcp.aip.org 


\title{
Nuclear quantum effects in electronically adiabatic quantum time correlation functions : Application to the absorption spectrum of a hydrated electron
}

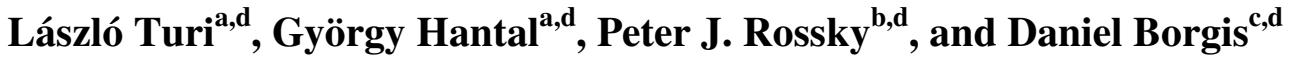 \\ ${ }^{a}$ Department of Physical Chemistry, Eötvös Loránd University, Budapest 112, P.O.Box 32, \\ Hungary, H-1518 \\ ${ }^{b}$ Department of Chemistry and Biochemistry and Institute for Computational Engineering and \\ Sciences, University of Texas at Austin, Austin, TX 78712-1167 \\ ${ }^{\mathrm{c}}$ Pôle de Chimie Théorique, UMR-CNRS PASTEUR, Ecole Normale Supérieure, 24, rue \\ Lhomond, 75231 Paris Cedex 05, France
}

\begin{abstract}
A general formalism for introducing nuclear quantum effects in the expression of the quantum time correlation function of an operator in a multi-level electronic system is presented in the adiabatic limit. The final formula includes the nuclear quantum time correlation functions of the operator matrix elements, of the energy gap, and their cross terms. These quantities can be inferred and evaluated from their classical analogs obtained by mixed quantum-classical molecular dynamics simulations. The formalism is applied to the absorption spectrum of a hydrated electron, expressed in terms of the time correlation function of the dipole operator in the ground electronic state. We find that both static and dynamic nuclear quantum effects distinctly influence the shape of the absorption spectrum, especially its high-energy tail related to transitions to delocalized electron states. Their inclusion does improve significantly the agreement between theory and experiment for both the low and high frequency edges of the spectrum. It does not appear sufficient, however, to
\end{abstract}

\footnotetext{
${ }^{\mathrm{d}}$ E-mail: turi@chem.elte.hu, fax: (36)-1-372-2592

hantal@elte.hu,fax: (36)-1-372-2592

rossky@mail.utexas.edu,fax: (1)-512-471-3555

daniel.borgis@ens.fr, fax: (33)-1-69-47-01-46.
} 
resolve persistent deviations in the slow Lorentzian-like decay part of the spectrum in the intermediate $2-3 \mathrm{eV}$ region. 


\section{Introduction}

Linear response theory makes it possible to relate experimental observables to time correlations functions (TCF), and, in general, to quantum time correlation functions (QTCF). It is of theoretical importance to be able to estimate these quantities. For many-body molecular systems, this implies knowing how to compute TCF's using molecular simulations. The quantum character can originate from the involvement of many electronic states, from quantum nuclear effects, or both. There is presently an intense activity in this field and several methods for computing quantum correlation functions have been proposed. For a fairly recent review, see, e.g., Ref. [1]. Those methods are devoted to either pure quantum nuclear dynamics $^{2,3,4,5,6,7,8,9,10}$ or multilevel electronic systems coupled to a classical or semiclassical bath. ${ }^{11,12,13,14,15}$ The ring polymer molecular dynamics method ${ }^{8}$ has also been extended to simulate the dynamics of electronic degrees of freedom with an application to a solvated electron in supercritical helium. ${ }^{16}$ Recently, Causo et al. ${ }^{14,15}$ have derived an adiabatic linearized path integral formula for quantum time correlation functions and they have applied their approach to the problem of electron transport in molten salt solutions. They considered the limit of classical nuclear motions.

In this paper we address the similar issue of electronically adiabatic QTCF's for cases when the nuclear modes should be quantized too. Our approach is inspired by a previous work where we derived a quantum time correlation formula for the nonadiabatic decay rate between two adiabatic quantum electronic states coupled to a nuclear bath starting from the Fermi golden rule. $^{17,18}$ The quantum time correlation of the nonadiabatic coupling operator, involving both electronic and nuclear degrees of freedom, was expressed in terms of the nuclear quantum time correlation functions of the state-to-state nonadiabatic (kinetic energy) coupling, of the energy gap, and of their cross terms. At the end, the transition rate can be 
computed from the classical nuclear counterpart of the correlation functions. Those are obtained using adiabatic mixed quantum-classical simulations, and they are further corrected to account for quantum effects according to various quantization schemes.

We applied the formalism to the nonadiabatic decay rate of an excited p-like hydrated electron to its s-like ground state and found that, depending on the quantization schemes applied, quantum corrections can change the classical transition rate dramatically. ${ }^{17}$ The present work follows a similar theoretical path. The theory will be illustrated by application to another unsolved problem concerning the hydrated electron: the influence of the static and dynamic nuclear quantum effects on its ground state absorption spectrum.

Since its original identification ${ }^{19}$ in 1962 the hydrated electron has been the subject of intensive experimental and theoretical research. As a result, structural, energetic and spectroscopic properties of the hydrated electron are understood in great detail. ${ }^{20}$ A longstanding issue, the shape of the optical absorption spectrum, however, is still not satisfactorily explained. Experiment measures a broad, featureless, asymmetric band with a maximum at $1.72 \mathrm{eV} .^{21}$ Theory, based mainly on a mixed quantum-classical approach, has not been able to fully describe the position and the shape, and particularly, the intensity of the blue-side of the spectrum, the high energy tail, with acceptable accuracy. ${ }^{22,23,24,25}$ Pseudopotential-based methods, although inadequate in some quantitative respects, shed light on fundamental qualitative aspects underlying the origin of the spectrum. It is accepted that three transitions dominate the spectrum, from the s-type electronic ground state to the first three, nondegenerate p-states. ${ }^{20}$ It was also found that solvent fluctuations determine the general shape and breadth of the optical spectra; radial fluctuations of the solvent cavity accomodating the electron influence the mean s-p energy gap, whereas fluctuations in the shape of the cavities (asymmetric distortions) modulate the splitting of the p-levels. ${ }^{20}$ The failure to correctly reproduce the experimental spectrum in simulations has two major, related origins. First, in 
most mixed quantum-classical approaches the solvent bath is treated classically, while the electron is described by quantum mechanics. The greater source of discrepancy between experiment and theory likely originates from the approximate nature of the pseudopotentials employed, especially in describing the energetics of the excited states. Subsequent improvements of the pseudopotentials led to a more satisfactory agreement of the position of the spectral maximum with experiment. ${ }^{25,26,27}$ Inclusion of solvent electronic polarizability makes it possible to fine tune the band maximum to the exact position. The high energy tail of the spectrum, however, is still not fully developed in any model. We note that the only ab initio molecular dynamics study of which we are aware on the bulk hydrated electron resulted in a similar characteristics of the absorption spectrum, with a correct position for the maximum but notable underestimation of the high energy tail. ${ }^{28}$

The effect of the quantum behavior of the solvent bath in calculated spectra, the second main source of discrepancy between experiment and theory, will be addressed here. The neglect of nuclear quantization is a problem that occurs when computing observables with any mixed quantum-classical approach, including available ab initio molecular dynamics techniques. A new approach to this general nuclear quantization issue will be the focus of the present paper.

The structure of the paper is as follows. In Sec. II we derive a theoretical expression for the quantum time autocorrelation function of an operator $\hat{A}$ acting on a multilevel electronic system coupled to a nuclear bath, in the electronically adiabatic limit, in terms of appropriate quantum correlation functions in nuclear space. These functions can be inferred from their classical counterparts, computed by classical molecular dynamics simulations, by using an appropriate quantization scheme. In Sec III, the theoretical approach is applied to the computation of the absorption spectrum, starting from the Gordon-Kubo formula. After providing the details of the simulations, we discuss the classical and quantized absorption 
spectra for the case of the hydrated electron, obtained according to our formalism, and evaluate the effects of various approximations. Sec. IV concludes the paper.

\section{Adiabatic quantum time correlation functions}

For a system described by a set of electronic states coupled to a bath of nuclear states, the thermally averaged time autocorrelation function of an operator $\hat{A}$ can be written as

$$
\langle\hat{A} \hat{A}(t)\rangle=\operatorname{Tr}\left[\hat{\rho}_{T} \hat{A} \hat{U}^{+}(t) \hat{A} \hat{U}(t)\right]=\sum_{I} \frac{e^{-\beta E_{I}}}{Z}\left\langle I\left|\hat{A} e^{+i \hat{H} t / \hbar} \hat{A} e^{-i H t / \hbar}\right| I\right\rangle
$$

where $\hat{\rho}_{T}$ is the canonical density operator, expanded on the basis of the eigenfunctions $|I\rangle$

of the full Hamiltonian of the system,

$$
\hat{\rho}_{T}=\frac{1}{Z} \sum_{I} e^{-\beta E_{I}}|I\rangle\langle I|
$$

with $Z=\operatorname{Tr}\left(e^{-\beta \hat{H}}\right)$, the canonical partition function, and $\hat{U}(t)=e^{-i \hat{H} t / \hbar}$ is the full quantum propagator of the system. In the Born-Oppenheimer approximation, when the state of the system can be expressed as a direct product of nuclear state $|p\rangle$ and electronic wave function $|i\rangle$, and assuming that only a single electronic state (the ground state) is accessible $(i=0)$ in the dynamic process, the time correlation function simplifies to

$$
\langle\hat{A} \hat{A}(t)\rangle=\sum_{p} \frac{e^{-\beta E_{p 0}}}{Z_{0}}\left\langle p\left|\left\langle 0\left|\hat{A} e^{+i \hat{H} t / \hbar} \hat{A} e^{-i \hat{H} t / \hbar}\right| 0\right\rangle\right| p\right\rangle=\left\langle\left\langle 0\left|\hat{A} e^{+i \hat{H} t / \hbar} \hat{A} e^{-i \hat{H} t / \hbar}\right| 0\right\rangle\right)
$$

In Eq (4) $Z_{0}$ is the canonical partition function for the thermal equilibrium of the nuclear modes, $p$, on the ground state electronic surface $|0\rangle$. In the following, for the sake of compactness, we will adopt the $\langle\ldots\rangle$ notation for thermal averaging (tracing) over the nuclear distribution on the ground state electronic surface, as implemented in the second equality of Eq. (3). Inserting the resolution of the identity for the electronic variables in terms of electronic states transforms Eq. (3) to 


$$
\begin{aligned}
\langle\hat{A} \hat{A}(t)\rangle & =\left\langle\sum_{k, l, m}\langle 0|\hat{A}| k\rangle\left\langle k\left|e^{+i \hat{H}_{t} / \hbar}\right| l\right\rangle\langle l|\hat{A}| m\rangle\left\langle m\left|e^{-i \hat{H}_{t} / \hbar}\right| 0\right\rangle\right\rangle \\
& =\sum_{k}\left\langle\langle 0|\hat{A}| k\rangle e^{+i \hat{H}_{k} t / \hbar}\langle k|\hat{A}| 0\rangle e^{-i \hat{H}_{0} t / \hbar}\right\rangle=\sum_{k}\left\langle A_{0 k} e^{+i \hat{H}_{k} t / \hbar} A_{k 0} e^{-i \hat{H}_{0} t / \hbar}\right\rangle
\end{aligned}
$$

Eq. (4) contains the matrix elements of operator $\hat{A}$, and the two nuclear Hamiltonians corresponding to the $0^{\text {th }}$ and $k^{\text {th }}$ Born-Oppenheimer potential surfaces. The expression can be easily modified to a form which is similar to that originally introduced by Staib and Borgis for the golden rule formula of non-adiabatic transition rates ${ }^{25}$

$$
\langle\hat{A} \hat{A}(t)\rangle=\sum_{k}\left\langle A_{0 k}^{(0)}(0) \exp _{(-)}\left[\frac{i}{\hbar} \int_{0}^{t} \Delta H_{k 0}^{(0)}(\tau) d \tau\right] A_{k 0}^{(0)}(t)\right\rangle
$$

where $\exp _{(-)}$is the negative time-ordered exponential, and $\Delta H_{k 0}^{(0)}(\tau)$ and $A_{k 0}^{(0)}(t)$ are defined as

$$
\Delta H_{k 0}^{(0)}(\tau)=e^{i H_{0} \tau \hbar}\left(\hat{H}_{k}-\hat{H}_{0}\right) e^{-i \hat{H}_{0} \tau \hbar},
$$

and

$$
A_{k 0}^{(0)}(t)=e^{i H_{0} t / \hbar}\langle k|\hat{A}| 0\rangle e^{-i \hat{H}_{0} t h} .
$$

The superscripts in Eqs. (5)-(7) indicate that the nuclear dynamics take place on the ground electronic surface. In the following we drop these superscripts for convenience.

To proceed forward to a more tractable form we follow a similar route to the one we applied in our previous paper on non-adiabatic transition rates. ${ }^{17}$ We use the following simple relation to bring an operator, $\hat{A}$, to exponential form:

$$
\hat{A}=\lim _{\lambda \rightarrow 0} \frac{\mathrm{d}}{\mathrm{d} \lambda} e^{\lambda \hat{A}},
$$

and then successively employ the cumulant expansion of the exponentials including the cumulant expansion of the time ordered exponential to second order. Since $\lambda$ is an arbitrary parameter, the order of differentiation with respect to $\lambda$ and tracing over the initial nuclear conditions can be exchanged. The final form of the time autocorrelation function reads now as 


$$
\begin{aligned}
\langle\hat{A} \hat{A}(t)\rangle= & \sum_{k=0}\left[e^{i\left(\Omega_{k 0}(t)\right\rangle t} \times \exp \left\{-\int_{0}^{t} d \tau_{1} \int_{0}^{\tau_{1}} d \tau_{2}\left\langle\delta \Omega_{k 0}(0) \delta \Omega_{k 0}\left(\tau_{2}\right)\right\rangle\right\} \times\left\{\left\langle A_{0 k}(0) A_{k 0}(t)\right\rangle\right.\right. \\
& +i\left\langle A_{0 k}\right\rangle \int_{0}^{t} \mathrm{~d} \tau\left\langle\delta \Omega_{k 0}(0) \delta A_{k 0}(\tau)\right\rangle+i\left\langle A_{k 0}\right\rangle \int_{0}^{t} \mathrm{~d} \tau\left\langle\delta A_{0 k}(0) \delta \Omega_{k 0}(\tau)\right\rangle \\
& \left.\left.-\int_{0}^{t} \mathrm{~d} \tau_{1} \int_{0}^{t} \mathrm{~d} \tau_{2}\left\langle\delta A_{0 k}(0) \delta \Omega_{k 0}\left(\tau_{1}\right)\right\rangle \times\left\langle\delta \Omega_{k 0}(0) \delta A_{k 0}\left(\tau_{2}\right)\right\rangle\right\}\right]
\end{aligned}
$$

where we introduced $\Omega_{k 0}(t)=\Delta H_{k 0}(t) / \hbar$, and $\delta$ stands for the fluctuations from the averages. This formula is reminescent of the adiabatic time correlation formula derived by Causo et al. from a linearized path-integral approach, followed by a cumulant expansion ${ }^{14,15}$ similar to ours. ${ }^{17}$ In their case, however, the dynamics appears to be defined on the half state $\frac{1}{2}(|0\rangle+|k\rangle)$ for each quantum number $k$ in the sum, whereas it is the ground state dynamics which emerges naturally for every $k$-state in our formalism.

At this stage, the time correlation functions appearing in Eq. (9) are fully quantum statistical objects in nuclear phase space and their direct evaluation remains a very difficult task. A second step can be taken by approximating those QTCF's from their classical counterparts using a suitable quantization scheme. Various formulae have been proposed to relate an arbitrary correlation function, $C^{q}(t)=\langle A(0) B(t)\rangle$, to its classical counterpart, $C(t) .{ }^{29,30,31,32,33,34,35,36} \mathrm{We}$ will argue below that, at least for the problem considered, the absorption spectrum of an excess electron in water, the so-called harmonic quantization scheme $e^{29,30}$ is adequate. The harmonic quantization, which is exact for harmonic systems, prescribes $\hat{C}^{q}(\omega)$ in the frequency domain as

$$
\hat{C}^{q}(\omega)=\frac{\beta \hbar \omega}{2 \tanh (\beta \hbar \omega / 2)} \times \frac{2}{1+e^{-\beta \hbar \omega}} \hat{C}(\omega)=\frac{\beta \hbar \omega}{1-e^{-\beta \hbar \omega}} \hat{C}(\omega)
$$

where $\hat{C}(\omega)$ is the Fourier transform of the classical correlation function $C(t), \hat{C}^{q}(\omega)$ is the quantized correlation function in the frequency domain. In the first equality, the first term 
accounts for the renormalization of the individual mode amplitudes when going from the classical to the quantum regime, whereas the second one accounts for the detailed balance condition fulfilled by quantum correlation functions, $\hat{C}^{q}(-\omega)=e^{-\beta \hbar \omega} \hat{C}^{q}(\omega)$. This formula transforms in the time domain to

$$
C^{q}(t)=\int_{0}^{\infty} d \omega \frac{\hat{C}(\omega)}{\pi} \frac{\beta \hbar \omega}{2}(\operatorname{coth}(\beta \hbar \omega / 2) \cos (\omega t)-i \sin (\omega t))
$$

For a multidimensional system, the determination of $C^{q}(t)$ implies the computation of the corresponding classical $C(t)$ by molecular dynamics simulations, its Fourier transform to get $\hat{C}(\omega)$, and then to return to the time domain using Eq. (11).

\section{Application to the absorption spectrum of a solvated electronic system}

\section{Theoretical expression of the absorption spectrum}

For a general electronic-bath quantum mechanical system, the frequency resolved absorption spectrum is given by the well-known Kubo-formula ${ }^{37}$

$$
I(\omega)=\frac{\omega}{2 \pi}(1-\exp (-\beta \hbar \omega)) \int_{-\infty}^{\infty} d t \exp (-i \omega t)\langle\hat{\mu} \hat{\mu}(t)\rangle
$$

where $\beta=1 / k T, \omega$ stands for the frequency, and $\hat{\mu}(t)$ denotes the time-dependent electronic dipole moment operator. $\mathrm{Eq}$ (12) contains the quantum time autocorrelation function of the dipole moment operator averaged over the electronic eigenstates and the solvent degrees of freedom.

Applying the general formula of Eq. (9) for $\hat{A}=\hat{\mu}$, we note first that the thermal average of the electric transition dipole moment $\mu_{0 k}$ disappears. Secondly, symmetry tells us that the trivial term of the sum $(k=0)$ should be equal to zero. Thus, for the time autocorrelation function of the dipole moment operator we have 


$$
\begin{aligned}
\langle\hat{\mu} \hat{\mu}(t)\rangle= & \sum_{k=1}\left[e^{i\left\langle\Omega_{k 0}\right\rangle t} \times \exp \left\{-\int_{0}^{t} d \tau_{1} \int_{0}^{\tau_{1}} d \tau_{2}\left\langle\delta \Omega_{k 0}(0) \delta \Omega_{k 0}\left(\tau_{2}\right)\right\rangle\right\} \times\left\{\left\langle\mu_{0 k}(0) \mu_{k 0}(t)\right\rangle\right.\right. \\
& \left.\left.-\int_{0}^{t} \mathrm{~d} \tau_{1} \int_{0}^{t} \mathrm{~d} \tau_{2}\left\langle\delta \mu_{0 k}(0) \delta \Omega_{k 0}\left(\tau_{1}\right)\right\rangle \times\left\langle\delta \Omega_{k 0}(0) \delta \mu_{k 0}\left(\tau_{2}\right)\right\rangle\right\}\right]
\end{aligned}
$$

Inserting Eq. (13) into Eq (12) one can easily obtain the frequency resolved absorption spectrum

$$
\begin{aligned}
& I(\omega)=\frac{\omega}{2 \pi}(1-\exp (-\beta \hbar \omega)) \times \\
& \times \sum_{k=1} \int_{-\infty}^{\infty} d t \exp \left(-i\left(\omega-\left\langle\Omega_{k 0}\right\rangle\right) t\right) \times \exp \left\{-\int_{0}^{t} d \tau_{1} \int_{0}^{\tau_{1}} d \tau_{2} C_{\Omega_{k 0}}^{q}(t)\right\} \times\left\{C_{\mu_{k 0}}^{q}(t)-\int_{0}^{t} \mathrm{~d} \tau C_{\mu_{k 0} \Omega_{k 0}}^{q}(\tau) \int_{0}^{t} \mathrm{~d} \tau C_{\Omega_{k 0} \mu_{k 0}}^{q}(\tau)\right\}
\end{aligned}
$$

where we have introduced an obvious notation for the quantum transition dipole moment correlation function, the frequency gap autocorrelation function, and their cross-correlation functions. Note again that the TCF's are still fully quantum objects at this stage.

Since, as mentioned earlier, the direct evaluation of the nuclear quantum correlation functions is a very difficult task, we opt for the usual alternative approach and replace the quantum correlation functions in a first step by their classical counterparts computed from mixed quantum-classical molecular dynamics simulations. For example, $\Omega_{k 0}(t)=\left(E_{k}(t)-E_{0}(t)\right) / \hbar$ is simply the time-dependent energy gap of the quantum subsystem submerged in the classical bath. A purely classical spectrum has the following form:

$$
\begin{aligned}
I^{c l}(\omega) & =\frac{\omega}{2 \pi}(1-\exp (-\beta \hbar \omega)) \times \\
& \times \sum_{k=1} \int_{-\infty}^{\infty} d t \exp \left(-i\left(\omega-\left\langle\Omega_{k 0}\right)\right) t\right) \times \exp \left\{-\int_{0}^{t} d \tau_{1} \int_{0}^{\tau_{1}} d \tau_{2} C_{\Omega_{k 0}}(t)\right\} \times\left\{C_{\mu_{k 0}}(t)-\left[\int_{0}^{t} \mathrm{~d} \tau C_{\mu_{k 0} \Omega_{k 0}}(\tau)\right]^{2}\right\}
\end{aligned}
$$

In the quantum version, Eq. (14), the TCF's have to be inferred from their classical analogs using a suitable quantization formula. We argued previously that the harmonic 
quantization scheme was fully justified for quantizing the solvated electron nonadiabatic transition rates. ${ }^{17,18}$ We have shown ${ }^{17}$ that, for the energy gap fluctuations, this scheme leads to the correct decoherence times for an excited p-electron when compared to the Gaussian packet propagation approach of Prezhdo and Rossky, ${ }^{38}$ and Turi and Rossky, ${ }^{39}$ both for water and methanol. This argument is still valid for the present application. We have also found that the non-adiabatic coupling fluctuations are strongly dominated by the librational and vibrational solvent modes. ${ }^{17,18}$ We will show in the next section that this is also true for the transition dipole fluctuations. A harmonic bath description (for which the formula is exact) is thus quite sensible, at least for this application.

Application to the hydrated electron: Mixed quantum-classical adiabatic molecular dynamics simulations

To compute the frequency resolved absorption spectrum of an equilibrium, ground state hydrated electron we have performed adiabatic mixed quantum-classical molecular dynamics simulations of a ground state electron embedded in a classical water bath. The basics of the method can be found in Ref. 40. The details of the actual simulations are similar to our previous simulation in Ref. 27. The solvent bath consists of 1600 water molecules in a cubic simulation cell, with a box length of $36.34 \AA$ A. The molecular interactions are described by a three-site classical model potential with added internal flexibility. The electron is treated quantum mechanically in a plane wave basis represented on $32^{3}$ gridpoints equidistantly distributed in the simulation box. The interaction between the quantum particle and the classical molecules is modeled by an electron-water molecule pseudopotential. ${ }^{27}$ The nuclear configurations are adiabatically propagated on the ground state potential surface using the sum of classical and Hellmann-Feynman forces. The long-range part of the potential and the forces are treated using a smooth cut-off. The simulation time step is $1 \mathrm{fs}$, and the simulation 
has been run for $25 \mathrm{ps}$. The transition dipole matrix elements were computed from direct quadrature in the position representation. The origin of the dipole vector was chosen to be the center of the simulation box. Since the excess electronic states are orthogonal, the choice of the dipole vector does not affect the integrals. To produce sufficiently small sampling intervals for the Fourier transforms, we proceeded in two different ways. First, we calculated the correlation functions in every $0.2 \mathrm{fs}$, using a linear interpolation between the simulated data points. This approach provides sufficient number of data points to produce smooth spectra, but may also lead to slight numerical artifacts. To rule out this possibility, for comparison, we have also fitted the dipole-dipole and gap autocorrelation functions as a sum of one Gaussian and one exponential function, and used the values of the analytic functions in the numerical procedure. For the evaluation of the time autocorrelation function of the dipole moment operator, we performed the summations in Eqs (9), (13)-(15) for the first twelve states of the hydrated electron in our model. The influence of higher states will also be discussed below.

\section{Classical spectrum}

Figure 1 shows the normalized classical gap and the transition dipole autocorrelation functions, $C_{\Omega_{k 0}}(t)$ and $C_{\mu_{k 0}}(t)$, respectively, for the first five $0 \rightarrow k$ transitions $(k=1-5)$. We notice that all functions decay quickly, reaching $<\sim 10 \%$ of their initial amplitude within the first 0.5 ps of the dynamics. Interestingly, the gap autocorrelation functions show very similar decay in time, irrespective of the width of the energy gap. This behavior reflects the fact that the fluctuations in the energy gaps take place largely in parallel fashion. The transition dipole autocorrelation functions have more distinct features. For the first three functions (electronic p-states), although some relatively minor differences appear in their decay, they show qualitatively similar behavior. On the other hand, the other functions, corresponding to higher 
transitions $(0 \rightarrow k$, where $k>3)$, have at least an order of magnitude smaller unnormalized amplitude and decay significantly faster than the first three functions. This indicates that the higher transitions contribute to the spectrum to a significantly lesser extent. The first one of these $(k=4)$ exhibits some oscillations due to water bending and stretching modes. All others for $k>5$ demonstrate a behavior similar to $k=5$, with a fast Gaussian-like decay and no apparent oscillations. The Fourier transform of the functions (see below) indicate that translational and librational modes dominate in the gap fluctuations and transition dipole correlations; higher frequency vibrations are essentially absent from the classical autocorrelation functions. As a very important further observation, we found that the (very noisy) cross-correlation terms appear to contribute negligibly in the classical treatment (see Eq. 15); the contribution remains $\sim 1 \%$ of that of the transition dipole autocorrelation function by the time the fast decaying gap correlation function drops below $1 \%$ of its original value.

In the next step, we have also calculated the classical absorption spectrum with and without the cross-correlation terms (see Eq. 15). The resulting spectra are practically indistinguishable. For this reason, we will not consider the cross-correlation terms in the remainder of the paper; that is, we use

$$
I^{c l}(\omega)=\frac{\omega}{2 \pi}(1-\exp (-\beta \hbar \omega)) \times \sum_{k=1} \int_{-\infty}^{\infty} d t \exp \left(-i\left(\omega-\left\langle\Omega_{k 0}\right\rangle\right) t\right) \times \exp \left\{-\int_{0}^{t} d \tau_{1} \int_{0}^{\tau_{1}} d \tau_{2} C_{\Omega_{k 0}}\left(\tau_{2}\right)\right\} \times C_{\mu_{k 0}}(t)
$$

The classical spectrum normalized to unity at its maximum is shown in Figure 2, with the subbands of the first eleven transitions. It is clear, that the three s-p transitions dominate the spectrum, all other contributions have significantly smaller weight. This observation is in agreement with the results of previous simulations of Rossky and his co-workers. ${ }^{20,22}$ Notable, nevertheless, is a distinct spectral high energy tail, observable from $2.5 \mathrm{eV}$ to $5 \mathrm{eV}$, resulting from higher transitions (see the inset in Fig. 2). Since the intensity of these transitions decays very slowly, the high energy tail extends through a long energy range. In an attempt to model 
the contribution of further possible high energy states to the spectrum we fit the decay of the maximum intensities of the bands for states $k=5-11$ by a Lorentzian function, and extrapolated the tendency for twelve more states $(k=12-23)$ (Fig. 3). We also assumed that all the bands of the higher states have the same Gaussian shape as the one we fitted for the last computed subband, $0 \square 11$, and their corresponding maxima (computed from the Lorentzian extrapolation) are separated by $0.07 \mathrm{eV}$. Fig. 2 also shows the classical spectrum augmented by the twelve extra states. Although the estimate is based on some ad hoc assumptions, it demonstrates the important role transitions to high energy, delocalized states can play in determining the spectral shape.

The computed spectrum based on the classical correlation functions can be compared to the experimental spectrum, ${ }^{21}$ as shown in Figure 4. The classically calculated spectrum estimates the position of the maximum of the experimental spectrum reasonably well $(1.90 \mathrm{eV}$ vs. $1.72 \mathrm{eV})$. We argued previously, that based on an estimate using dielectric theory arguments, the simulated peaks are expected to be slightly red-shifted (by about $0.2-0.3 \mathrm{eV}$ ), and, thus, in better agreement with the measurements, after the proper self-consistent treatment of solvent electronic polarization in the presence of the excited electronic state..$^{27,41}$ The width of the optical band at half of its maximum, $0.73 \mathrm{eV}$, also compares favorably to the experimental $0.85 \mathrm{eV}$. The underestimation is mainly due to the fact that the classically computed spectrum suffers from a well-known deficiency; it underestimates the high energy tail of the absorption band. Nevertheless, due to the acceptable reproduction of the position of the maximum and the appearance of the higher energy spectral feature, the present classical results represent clear improvement relative to previous results. It is interesting to compare the classical spectrum computed in the present work to our previously published spectrum ${ }^{27}$ based on the histogram technique calculation of the transition dipole matrix elements in the slow-modulation limit. ${ }^{25}$ Figure 4 illustrates that the spectra appear reasonably similar, but the 
full classical treatment (Eq. 16) results in broader, more developed spectrum than in the previous approximate procedure.

\section{Quantized spectra}

We computed the quantized spectra using the quantized form of Eq. (16),

$$
I^{q}(\omega)=\frac{\omega}{2 \pi}(1-\exp (-\beta \hbar \omega)) \times \sum_{k=1} \int_{-\infty}^{\infty} d t \exp \left(-i\left(\omega-\left\langle\Omega_{k 0}\right\rangle\right) t\right) \times \exp \left\{-\int_{0}^{t} d \tau_{1} \int_{0}^{\tau_{1}} d \tau_{2} C_{\Omega_{k 0}}^{q}\left(\tau_{2}\right)\right\} \times C_{\mu_{k 0}}^{q}(t)
$$

where the $q$ superscript indicates the quantized quantities. In the quantization procedure we followed the harmonic quantization scheme ${ }^{29,30}$ according to Eq. (11). Quantum corrections apparently influence the autocorrelation functions, increasing the individual mode amplitudes, as shown in Fig 5 for the harmonic quantization scheme. Although it is still the translational and librational modes which dominate the quantized spectra, as they did the classical result, the contributions of the higher frequency vibrational modes are notably increased. As a further illustration of the influence of the quantum corrections, one can directly compare the $t=0$ values of the classical and quantum corrected autocorrelation functions. These values are collected in Table I, with the corresponding quantum to classical ratios. The initial value of the gap autocorrelation functions, which appears in the exponential of Eqs (16) and (17), and influences the width of the individual absorption bands, increases by a consistent $60-70 \%$ after quantization. It is clear, however, from Eqs (16) and (17) that the dipole moment autocorrelation function bears a more direct role in determining the intensity of the spectrum. In this respect, we take notice of two effects. First, the initial value of the transition dipole moment autocorrelation function drops by a factor of $\sim 20$ going from $k=3$ to $k=4$ so that the high energy states contribute to the spectrum to a significantly lesser extent. On the other hand, we observe that the effect of quantum corrections appear more important for higher $k$ values, gradually approaching a ratio of $\sim 5$ for the highest computed transitions. This 
indicates that quantum effects clearly play an important role in influencing the shape of the spectrum, especially at its high energy tail and mainly through the transition dipole moment correlation functions.

In computing the spectrum, in parallel to our previous work, ${ }^{17}$ we may assume that since solvent dynamics occurs on a slower timescale, the exponential function of the gap autocorrelation function can be replaced by a Gaussian function, the dephasing function.

$$
\exp \left\{-\int_{0}^{t} d \tau_{1} \int_{0}^{\tau_{1}} d \tau_{2} C_{\Omega_{k 0}}^{q}\left(\tau_{2}\right)\right\}=\exp \left\{-\frac{1}{2}\left\langle\delta \Omega_{k 0}^{2}\right\rangle^{q} t^{2}\right\}
$$

In the harmonic quantization this approximation leads to the following equation:

$$
\left\langle\delta \Omega_{k 0}^{2}\right\rangle^{q}=\left\langle\delta \Omega_{k 0}^{2}\right\rangle \int_{0}^{\infty} d \omega \frac{\tilde{C}_{\Omega_{k 0}}(\omega)}{\pi} \frac{\beta \hbar \omega}{2} \operatorname{coth}\left(\frac{\beta \hbar \omega}{2}\right)
$$

where $\tilde{C}_{\Omega_{k 0}}(\omega)$ is the normalized classical gap autocorrelation function. Obviously, one can perform the full evaluation of the double integral of the gap autocorrelation function without difficulty. We find that the results with the Gaussian approximation and the full calculation are very similar, demonstrating that the Gaussian approximation works reasonably well for the computation of the quantized spectra. The fully corrected spectrum computed with the double integral, is shown in the upper panel of Figure 6, while in the lower panel the maxima of the computed spectra were linearly shifted to match the maximum of the experimental spectrum, $1.72 \mathrm{eV}$. Accordingly, the application of the harmonic quantization scheme results in a small, but visible, quantum effect on the position of the spectrum, as the maximum shifts from $1.90 \mathrm{eV}$ to $1.87 \mathrm{eV}$. The half-width also increases very slightly to $0.74 \mathrm{eV}$ (from 0.73 $\mathrm{eV}$ ). More significant changes are apparent in the increased intensity of the high energy side of the spectrum. The intensity of the high energy tail of the classical spectrum at $3.0 \mathrm{eV}$ is only $\sim 3 \%$ of the intensity of the maximum; this value basically doubles to approximately $\sim 7 \%$ in the harmonic quantization scheme. Based on our observation of the dependency of the classical spectrum on the number of high energy states (i.e. Figs. 2 and 3), one can anticipate 
that inclusion of additional higher energy transitions will further improve the agreement between the computed and the experimental data on the blue side of the spectrum. Another promising aspect of the harmonic quantized spectrum is observable at the low energy side of the spectrum. Here, Fig. 6 reveals that the quantization notably improves the shape of the spectrum at $\sim 1 \mathrm{eV}$; the quantized spectrum reproduces the shape of the experimental spectrum at lower energies well.

\section{Discussion and Conclusions}

We have introduced a quantum mechanical formula for time autocorrelation functions of an operator $\hat{A}$ in the adiabatic (Born-Oppenheimer) limit. The formula is based on the application of the second order cumulant expansion of the exponential operator. It involves the nuclear quantum correlation functions of the operator matrix elements, the energy gap fluctuations, and the cross terms. Those quantum statistical objects can be approximated from their classical counterparts by suitable relations in frequency space. The corresponding expression for the particular case of the absorption spectrum, related to the dipole quantum autocorrelation function through the Kubo formula, involves the nuclear quantum time correlation functions of the energy gap, of the electronic transition dipole matrix elements, and their cross-terms.

These quantities were evaluated from mixed quantum-classical molecular dynamics simulations of a hydrated electron equilibrated in its ground state. The absorption spectrum computed from the resulting classical functions predicts the position of the maximum reasonably well and also possesses a high energy tail which is, however, not sufficiently developed compared to experiment. In addition, we pointed out that the high energy tail of the classical spectrum converges slowly with inclusion of additional high energy transitions, and 
so this comparison may improve with a more complete treatment of high energy states. The classical spectrum is smoother, and slightly broader, than computed previously with a histogram evaluation of the transition dipole matrix elements. The quantum corrections provide further important improvements. The harmonic quantization procedure, that we argued to be quite reasonable in this instance, significantly influences the spectral shape. First, it shifts the spectrum slightly to the red, and broadens it by $0.01 \mathrm{eV}$. More substantial changes, are observed at the wings of the optical band. The red side of the spectrum improves significantly relative to the experimental shape, while the large-energy tail gains intensity, approximately doubling that of the classical spectrum. Nevertheless, it is apparent that, even with such improvements, the quantized spectrum is still distinctly lacking in intensity at around $2.5 \mathrm{eV}$. One likely source is an inadequacy of the present pseudopotential when describing the higher lying, delocalized, excited states of the hydrated electron system. However, it is quite interesting to note that a recent application of the same pseudopotential to the case of negatively charged water clusters ${ }^{42}$ does yield a spectral shape which includes the high energy tail in at least qualitative agreement with the tail seen experimentally for the absorption spectra of clusters. ${ }^{43}$ For these cases, the excess electron is bound to the cluster surface in simulations, and the higher energy states contributing to this tail are apparently relatively asymmetric compared to the bulk water solvated states. We believe that this observation may provide the clue to resolving this persistent discrepancy in lineshape between simulated and experimental hydrated electrons.

The purpose of the present work was, however, not to reproduce the absorption spectrum of the hydrated electron system per se, but rather to illustrate a general formulation of adiabatic quantum time correlation functions for electrons/nuclei systems that provides a straightforward, although approximate, way to include nuclear quantum effects. The chosen 
example does emphasize that those effects can indeed be important and that their inclusion provides a significant improvement for the computation of a time-dependent observable.

\section{Acknowledgments}

L.T. gratefully acknowledges the tenure of the Öveges Research Fellowship. The work was supported by grants to L. T. from the National Research Fund of Hungary (OTKA) under Contracts No. T049715, IN69707 and K75285. P. J. R. is grateful to the National Science Foundation (CHE-0615173) and the R. A. Welch Foundation (F-0019) for support of this work. 
Table I. The initial $t=0$ value of the classical gap-gap and transition dipole moment autocorrelation functions, and their quantum/classical ratios.

\begin{tabular}{ccccc}
\hline $\begin{array}{c}\text { 0पk transition } \\
k\end{array}$ & $\left\langle\delta \Omega_{k 0}^{2}\right\rangle / \mathrm{fs}^{-2}$ & $\left\langle\delta \Omega_{k 0}^{2}\right\rangle^{q}$ & $C_{\mu_{k 0}}^{q}(0) /\left(e^{2} \mathrm{bohr}^{2}\right)$ & $C_{\mu_{k 0}}^{q}(0) / C_{\mu_{k 0}}(0)$ \\
\hline 1 & 0.07280 & 1.46 & 7.676 & 1.32 \\
2 & 0.06469 & 1.50 & 6.540 & 1.55 \\
3 & 0.07134 & 1.55 & 5.377 & 1.30 \\
4 & 0.1338 & 1.67 & 0.2007 & 1.89 \\
5 & 0.1571 & 1.65 & 0.0890 & 2.68 \\
6 & 0.1589 & 1.67 & 0.0690 & 3.86 \\
7 & 0.1631 & 1.68 & 0.0595 & 4.44 \\
8 & 0.1674 & 1.69 & 0.0555 & 4.78 \\
9 & 0.1720 & 1.68 & 0.0512 & 5.04 \\
11 & 0.1783 & 1.69 & 0.0485 & 5.05 \\
\hline & 0.1853 & 1.70 & 0.0468 & 4.72 \\
\hline
\end{tabular}




\section{Figure Captions}

Figure 1. Normalized classical gap and transition dipole autocorrelation functions, $C_{\Omega_{k 0}}(t)$ and $C_{\mu_{k 0}}(t)$, respectively, for the first five $0 \rightarrow k$ electronic transitions $(k=1-5)$. Solid line: $k=1$, dash: $k=2$, dot: $k=3$, dash-dot: $k=4$, dash-dot-dot $k=5$.

Figure 2. The absorption spectrum of an equilibrium, ground state hydrated electron computed using the classical correlation functions, Eq. (16). The spectrum is normalized to unity at its maximum. The inset shows all computed spectral contributions to the high energy tail. The figure also includes the extrapolated spectrum (dash) including the contributions from higher energy $0 \rightarrow k$ bands $(k=12-23)$.

Figure 3. The absorption bands of the highest seven computed $0 \rightarrow k$ transitions $(k=5-11)$, with a fitted Lorentzian function to model the progression of the band maxima of higher energy delocalized states.

Figure 4. Comparison of the absorption spectrum of an equilibrium, ground state hydrated electron computed using the classical correlation functions (solid line) to the experimental curve (dash), ${ }^{21}$ and the previously published spectrum in the slow-modulation limit (dotted line). ${ }^{27}$ The figure also includes the extrapolated spectrum (dashed) including the contributions from higher energy $0 \rightarrow k$ bands $(k=12-23)$.

Figure 5. Fourier transforms of the gap (left) and the transition dipole autocorrelation functions (right) for the $0 \rightarrow 1$ (upper panels) and $0 \rightarrow 5$ transitions (lower panels). The dashed line corresponds to the classical function, while the solid line shows the quantized correlation 
functions using the harmonic quantization scheme. Note the different scales between the two frames showing the transition dipole autocorrelation functions.

Figure 6. The absorption spectrum of an equilibrium, ground state hydrated electron computed using classical correlation functions (dash-dot) and the harmonic quantization scheme $\mathrm{e}^{29,30}$ (dashed). The spectra are normalized to unity at their maximum. The experimental spectrum is shown for comparison (solid line). ${ }^{21}$ The absolute peak intensities (Eq. 12) are 50.9 a.u. and 60.5 a.u. for the computed classical and the quantized spectra, 32.8 a.u. for the experiment (corresponding to the maximum molar absorption coefficient, $22700 \mathrm{dm}^{3} \mathrm{~mol}^{-1} \mathrm{~cm}^{-}$ $\left.{ }^{1}\right) .{ }^{44}$ The lower panel shows the calculated spectra linearly shifted to align the maximum with the experimental spectrum, $1.72 \mathrm{eV}$. 
Figure 1. Turi, Hantal, Rossky, and Borgis
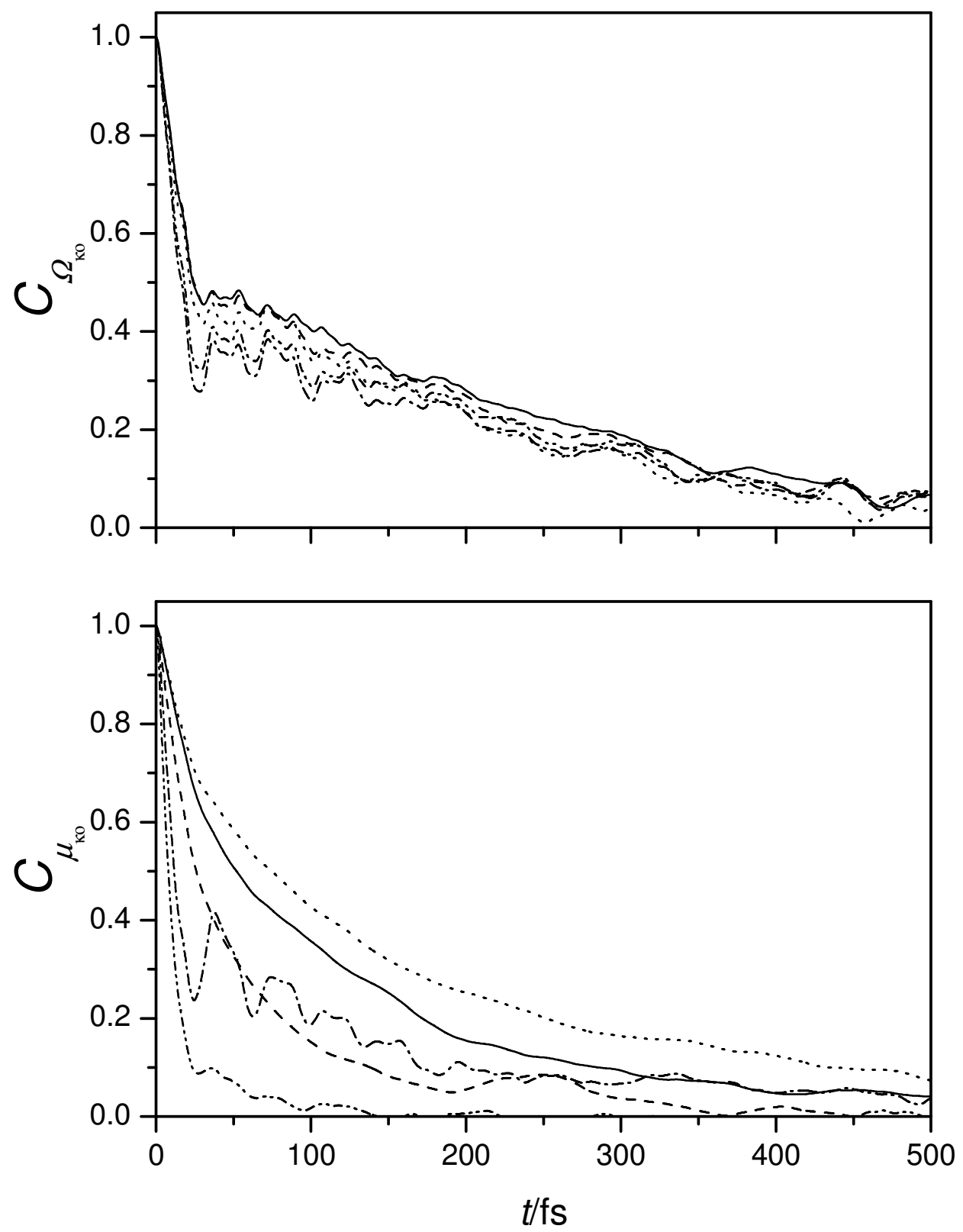
Figure 2. Turi, Hantal, Rossky, and Borgis

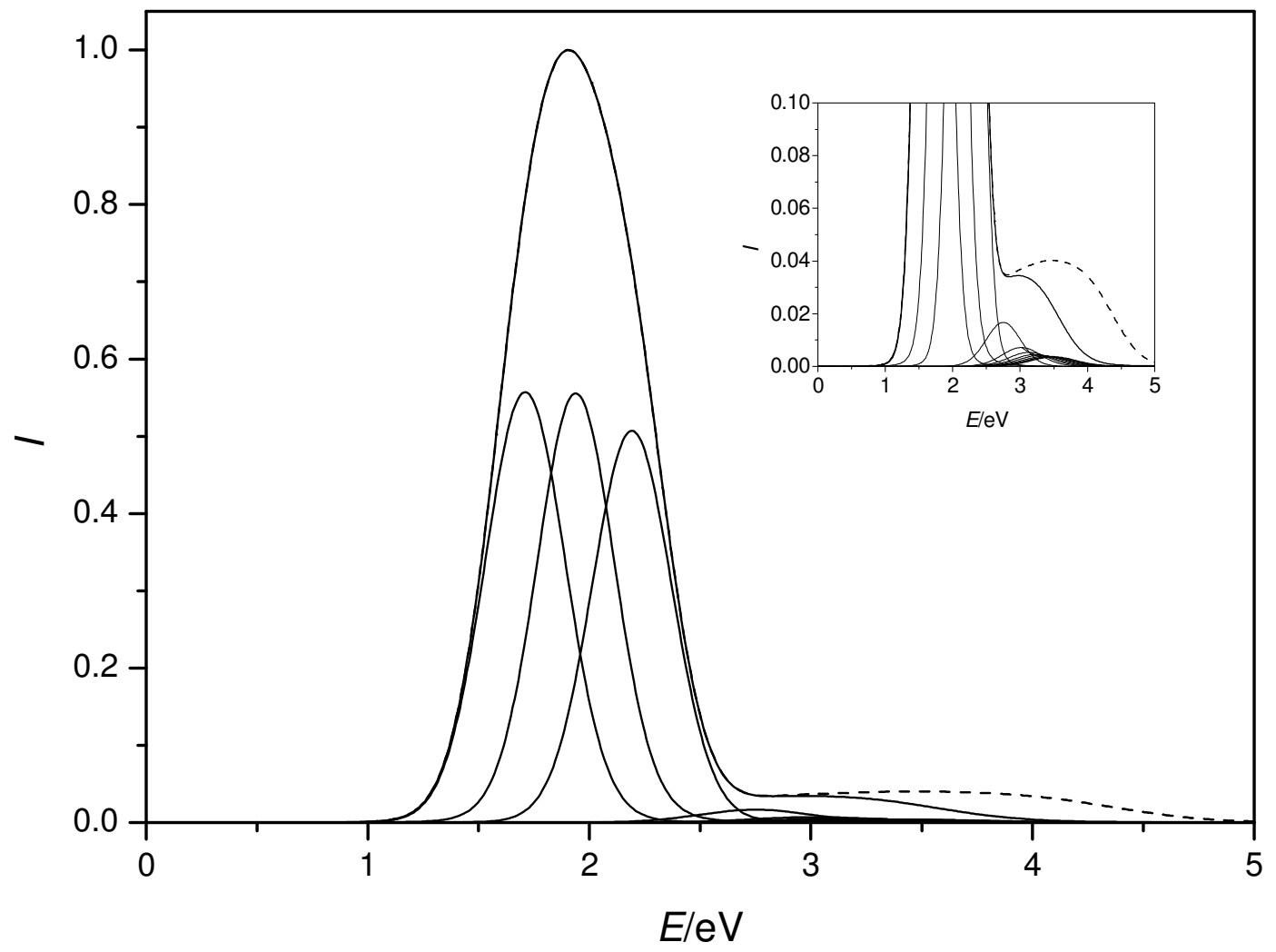


Figure 3. Turi, Hantal, Rossky, and Borgis

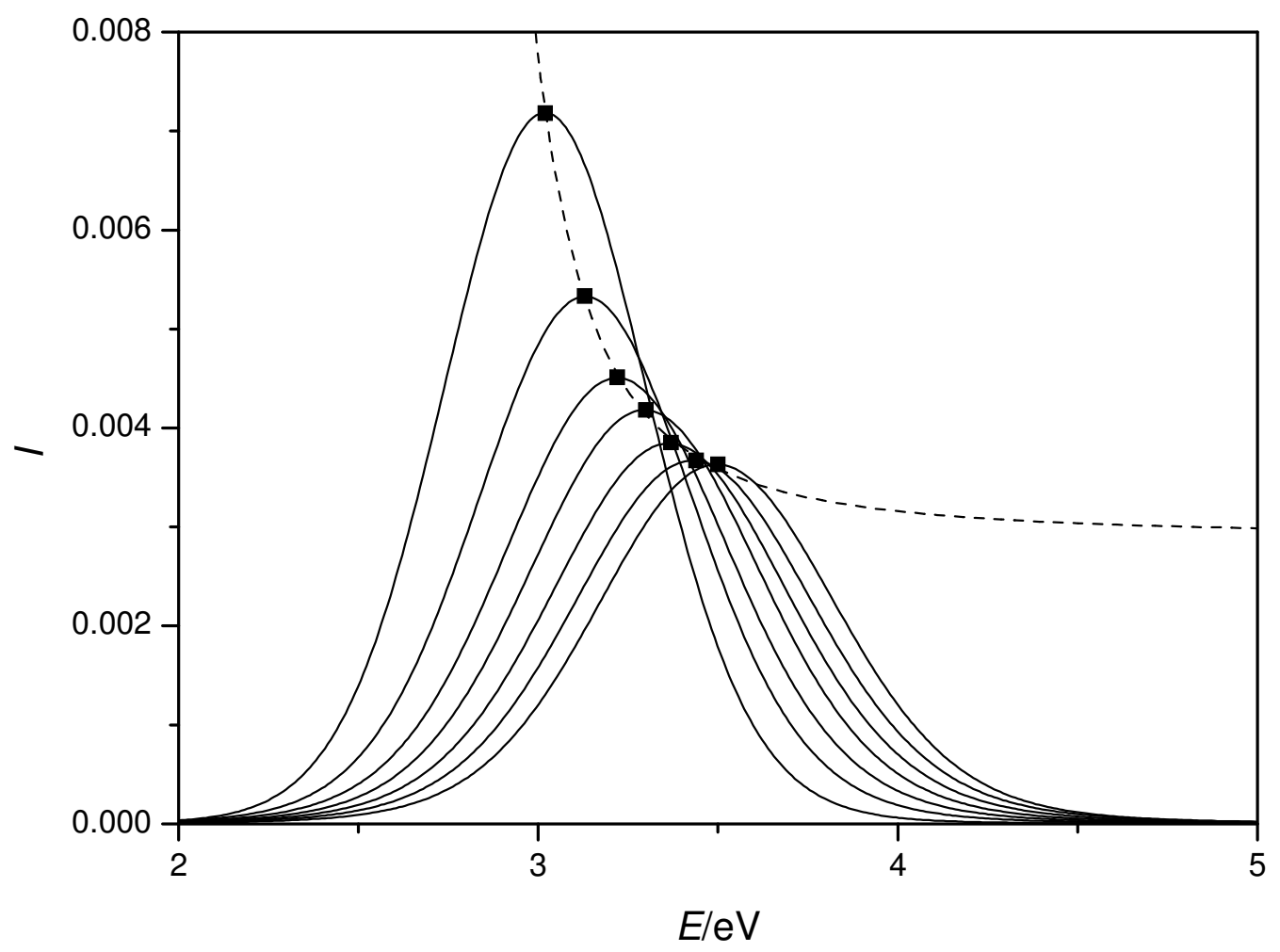


Figure 4. Turi, Hantal, Rossky, and Borgis

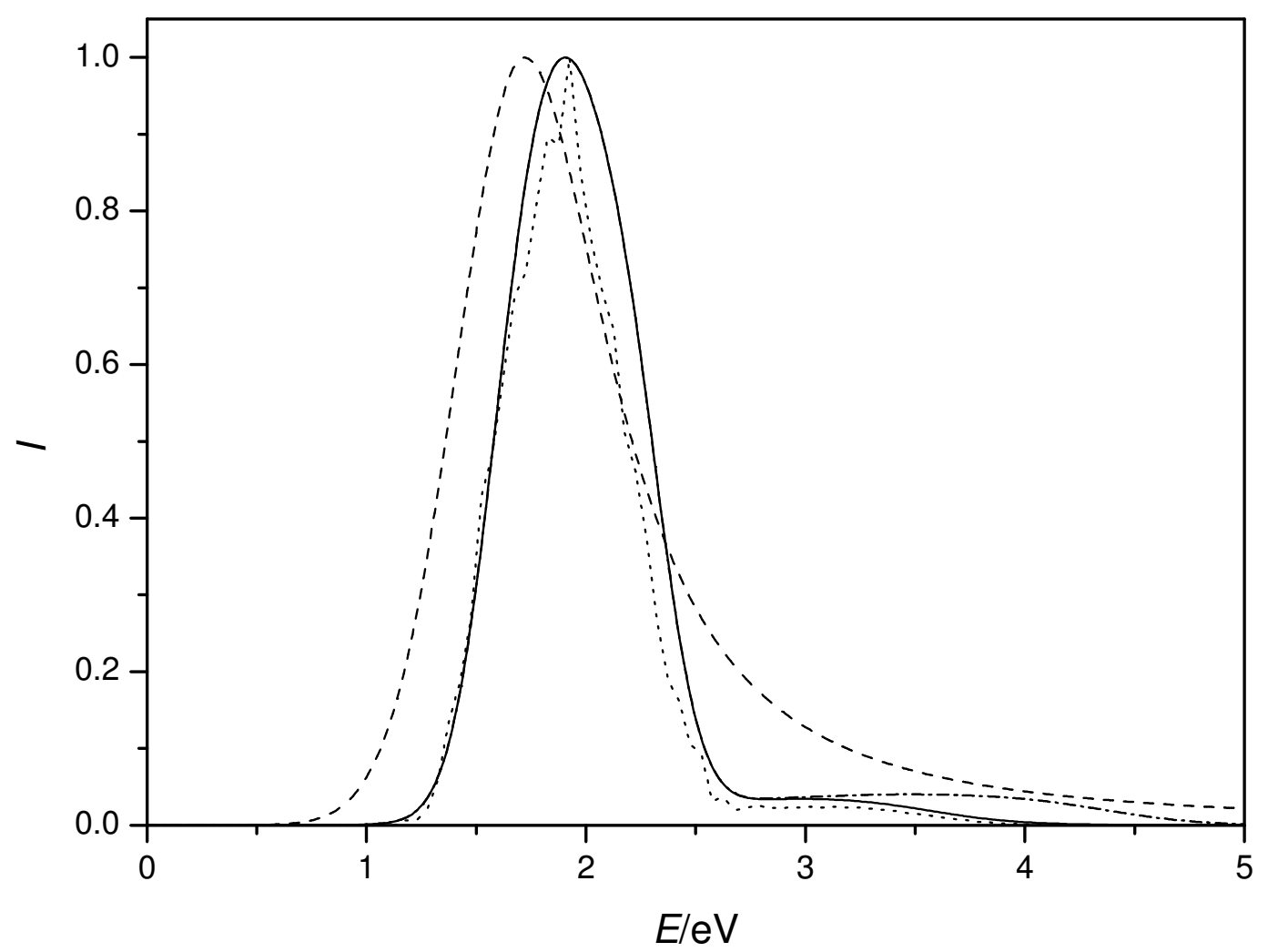


Figure 5. Turi, Hantal, Rossky, and Borgis
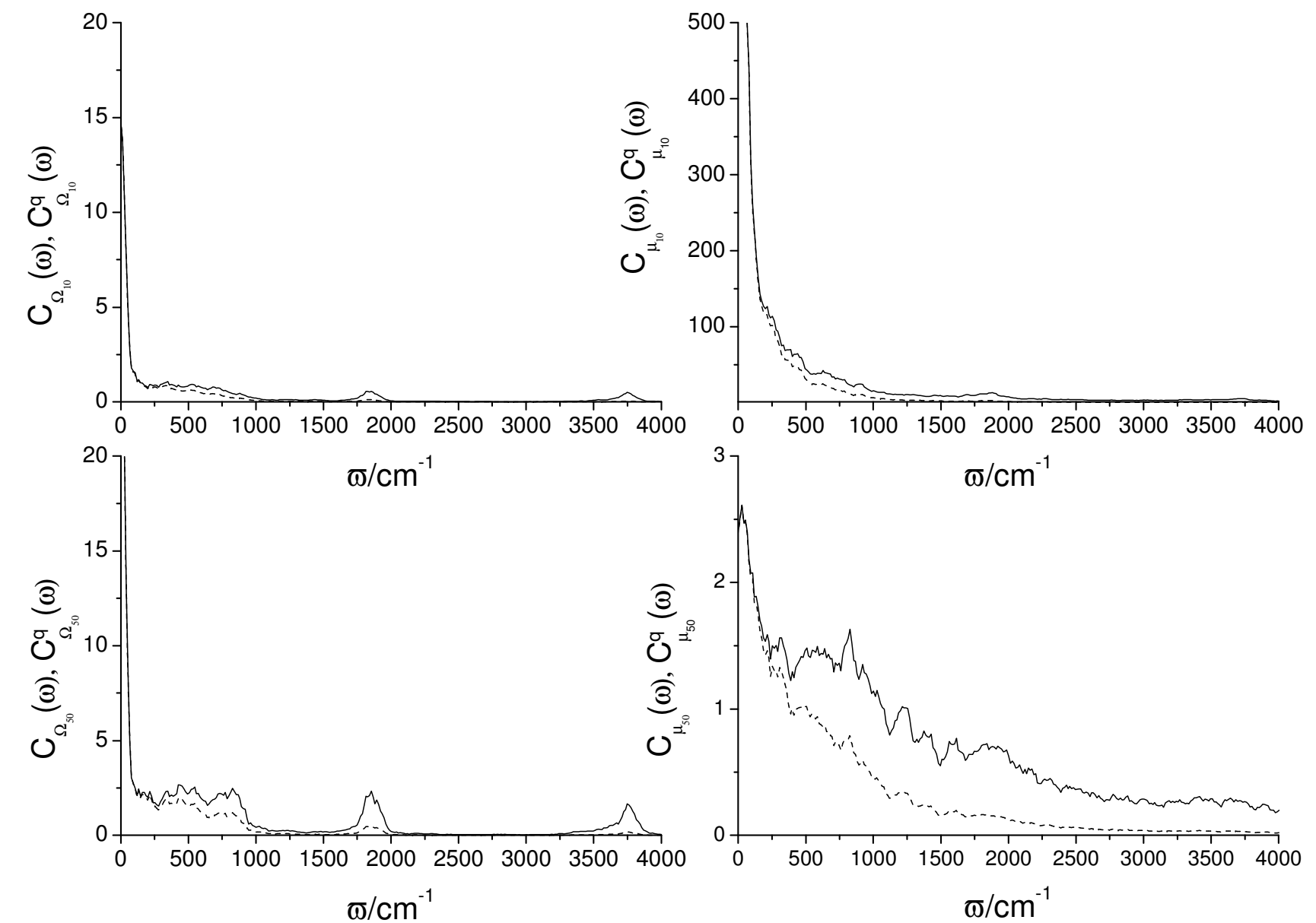
Figure 6. Turi, Hantal, Rossky, and Borgis
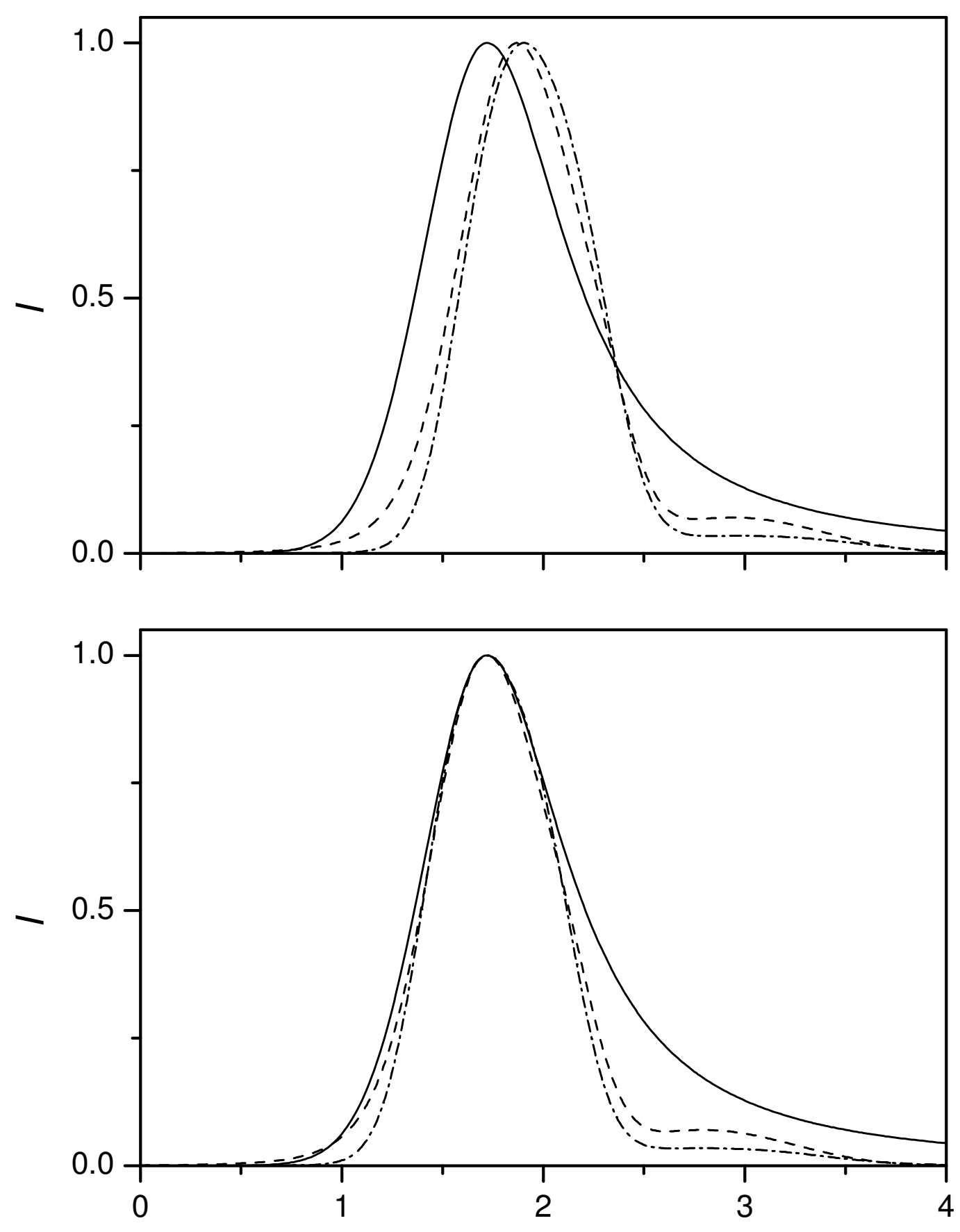

$\mathrm{E} / \mathrm{eV}$ 


\section{References}

${ }^{1}$ G. Stock and M. Thoss, Adv. Chem. Phys. 131, 243 (2005).

2 J. A. Poulsen, G. Nyman, and P. J. Rossky, J. Chem. Phys. 119, 12179 (2003).

${ }^{3}$ J. A. Poulsen, G. Nyman, P. J. Rossky, PNAS 102, 6709 (2005).

${ }^{4}$ J. A. Poulsen, G. Nyman, P. J. Rossky, J. Chem. Theor. Comp. 2, 1482 (2006).

${ }^{5}$ Q. Shi and E. J. Geva, J. Chem. Phys. 118, 8173 (2003).

${ }^{6}$ G. Krilov, E. Sim, and B. J. Berne, J. Chem. Phys. 114, 1075 (2001).

${ }^{7}$ V. Krishna and G. A. Voth, J. Phys. Chem. B 110, 18953 (2006).

${ }^{8}$ I. R. Craig and D. E. Manolopoulos, J. Chem. Phys. 121, 3368 (2004).

${ }^{9}$ X. Sun, H. Wang, and W. H. Miller, J. Chem. Phys. 109, 7064 (1998).

${ }^{10}$ H. Wang, M. Thoss and, W. H. Miller, J. Chem. Phys. 115, 2979 (2001).

${ }^{11}$ N. Makri, J. Phys. Chem. A 108, 806 (2004).

${ }^{12}$ J. Kegerreis and N. Makri, Chem. Phys. Lett. 467, 430 (2009).

${ }^{13}$ S. Bonella and D. Coker, Comp. Phys. Comm. 169, 267 (2005).

${ }^{14}$ M. S. Causo, G. Ciccotti, D. Montemayor, S. Bonella, and D. F. Coker, J. Phys. Chem. B 109, 6855 (2005).

${ }^{15}$ M. S. Causo, G. Ciccotti, S. Bonella, and R. Vuilleumier, J. Phys. Chem. B 110, 16026 (2006).

${ }^{16}$ T. F. Miller, III. J. Chem. Phys. 129, 194502 (2008)

${ }^{17}$ D. Borgis, P. J. Rossky, and L. Turi, J. Chem. Phys. 125, 064501 (2006).

${ }^{18}$ D. Borgis, P. J. Rossky, and L. Turi, J. Chem. Phys. 127, 174508 (2007).

${ }^{19}$ E. J. Hart and J. W. Boag, J. Am. Chem. Soc. 84, 4090 (1962).

${ }^{20}$ P. J. Rossky and J. Schnitker, J. Phys. Chem. 92, 4277 (1988).

${ }^{21}$ F.-Y. Jou and G. R. Freeman, J. Phys. Chem. 81, 909 (1977).

${ }^{22}$ J. Schnitker, K. Motakabbir, P. J. Rossky and R. Friesner, Phys. Rev. Lett. 60, 456 (1988). 
${ }^{23}$ A. Wallqvist, G. Martyna and B. J. Berne, J. Phys. Chem. 92, 1721 (1988).

${ }^{24}$ R. N. Barnett, U. Landman and A. Nitzan, J. Chem. Phys. 89, 2242 (1988).

${ }^{25}$ A. Staib and D. Borgis, J. Chem. Phys. 103, 2642 (1995).

${ }^{26}$ L. Turi, M.-P. Gaigeot, N. Levy and D. Borgis, J. Chem. Phys. 114, 7805 (2001).

${ }^{27}$ L. Turi and D. Borgis, J. Chem. Phys. 117, 6186 (2002).

${ }^{28}$ M. Boero, M. Parrinello, K. Terakura, T. Ikeshoji, and C. C. Liew, Phys. Rev. Lett. 90, 226403 (2003).

${ }^{29}$ P. H. Berens, S. R. White, and K. R. Wilson, J. Chem. Phys. 75, 515 (1981).

${ }^{30}$ J. S. Bader and B. J. Berne, J. Chem. Phys. 100, 8359 (1994).

${ }^{31}$ B. J. Berne and G. D. Harp, Adv. Chem. Phys. 17, 63 (1970).

${ }^{32}$ D. W. Oxtoby, Adv. Chem. Phys. 47, 487 (1981).

${ }^{33}$ P. Schofield, Phys. Rev. Lett. 4, 239 (1960).

${ }^{34}$ P. A. Egelstaff, Adv. Phys. 11, 203 (1962).

${ }^{35}$ K. F. Everitt, S. A. Egorov, and J. L. Skinner J. Phys. Chem. B 103, 9494 (1999).

${ }^{36}$ H. Kim and P. J. Rossky J. Phys. Chem. B 106, 8240 (2002).

${ }^{37}$ R. Kubo, J. Math. Phys. 4, 174 (1963).

${ }^{38}$ O. V. Prezhdo and P. J. Rossky, J. Chem. Phys. 107, 5863 (1997).

${ }^{39}$ L. Turi and P. J. Rossky, J. Chem. Phys. 120, 3688 (2004).

${ }^{40}$ F. A. Webster, P. J. Rossky, and R. A. Friesner, Comput. Phys. Commun. 63, 494 (1991).

${ }^{41}$ D. Borgis and P. J. Rossky, private communications.

${ }^{42}$ Á. Madarász, P. J. Rossky, and L. Turi, J. Chem. Phys. 130, 124319 (2009).

${ }^{43}$ P. Ayotte, and M. A. Johnson, J. Chem. Phys. 106, 811 (1997).

${ }^{44}$ P. M. Hare, E. A. Price, and D. M. Bartels, J. Phys. Chem. A 112, 6800 (2008). 